\title{
Potencial Anti-inflamatory Effects of Artemisia Gorgonum on Rat Liver Injury Induced by $\mathrm{CCl}_{4}$
}

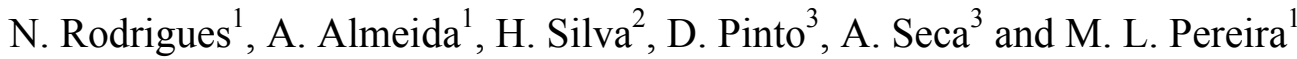 \\ 1. Dep. of Biology \& CICECO - Aveiro Institute of Materials University of Aveiro, Campus de \\ Santiago, 3810-193 Aveiro, Portugal. \\ 2. Dep. of Biology \& CESAM, University of Aveiro, Campus Santiago, 3810-193 Aveiro, Portugal. \\ 3. Dep. of Chemistry \& QOPNA, University of Aveiro, Campus Santiago, 3810-193 Aveiro, Portugal.
}

Artemisia gorgonum (Asteraceae) is a common traditional Cape Verde medicinal plant which has been used intensively to treat several inflammatory diseases. However, the most recent studies reported the antimalaria activity ${ }^{[1]}$, antiviral, anti-tumoral, anti-pyretic, anti-hemorrhagic, anti-coagulant, anti-fungal, antimicrobial, anti-oxidant, anti-malarial, anti-ulcerogenic and antispasmodic and hepatoprotective activities have been reported for the Artemisia genus ${ }^{[1-5]}$. Therefore, our aim is to investigate the protective effect of aqueous extract of $A$. gorgonum (AEAG) against liver injury on rat model.

The inflorescences AEAG were extracted in $80 \%$ ethanol during two days by rotary evaporator extraction at the boiled temperature. After filtration, the aqueous extract was dried by a vacuum and other parts were lyophilized.

Male wistar rats were divided randomly into three groups ( $\mathrm{n}=5$ each): first group (normal control) given orally $0.5 \% \mathrm{CMC}$ (extract suspended in $0.5 \%$ sodium carboxymethyl cellulose) for 7 days and then intraperioneally injected with $10 \mathrm{ml} / \mathrm{kg} /$ body weight olive oil. The second group (hepatotoxicity control) was orally given $0.5 \%$ of CMC for seven days and then i.p. injected with $\mathrm{CCl}_{4}(0.1 \%$ in olive oil, $10 \mathrm{ml} / \mathrm{kg}$, i.v ). The third group was pretreated with AEAG at only dose $(200 \mathrm{mg} / \mathrm{kg}$ per day p.o) during the same period and then hepatotoxicity was induced, by $\mathrm{CCl}_{4}(0.1 \%$ in olive oil, $10 \mathrm{ml} / \mathrm{kg})$ i.p. injected ${ }^{[1]}$. On the final experimental day, after $24 \mathrm{~h}$ all the animals were weighed and sacrificed. The liver tissues fixed in Bouin's solution were embedded in paraffin and sectioned into $5 \mu \mathrm{ml}$ sections for histomorphological analysis. Section slides were stained with hematoxylin and eosin (H\&E) and Masson's trichrome. Observations and photographs were made using a digital camera (Olympus Camedia C-5060) attached to a microscope (Olympus BX41, Tokyo, Japan). Experiments were conducted based on ethical and scientific principles for animal testing.

The results showed several changes on hepatic sections from second group who were administrated with $\mathrm{CCl}_{4}$ only (hapatotoxicity control). Histopathological analysis indicated hepatocellular necrosis, ballooned hepatocytes, vacuolation, hemorrhage at portal vein, and bridging collagen accumulation in the $\mathrm{CCl}_{4}$ group, whereas AEAG treatment evidently alleviated these features (Fig. 1A and B).

Our results demonstrated that aqueous extract of $A$. gorgonum strongly indicate the hepato-protective against liver injury induced by $\mathrm{CCl}_{4}$ which may be attributed to its immunomodulatory or antioxidative activity, and thereby scientifically supports the traditional use [6]. 


\section{References:}

[1] N. Amat et al., Journal of Ethnopharmacol., 131(2) (2010) p. 478.

[2] R. Ortet et al., Phytochem. 69(17) (2008) p. 2961.

[3] S. Rashid et al., Food Chem. 138(1) (2013) p. 693.

[4] P. Remberg et al., Phytomedicine 11(1) (2004) p. 36.

[5] R.X. Tan et al., Planta Med 64(04) (1998) p. 295.

[6] Funding for this work was provided by CICECO - Aveiro Institute of Materials University of Aveiro (Portugal), Pest-C/CTML/LA0011/2011-FCT. Thanks are due to Fundação para a Ciência e a Tecnologia (FCT, Portugal), European Union, QREN, FEDER and COMPETE for funding the QOPNA research unit (project PEst-C/QUI/UI0062/2011).

Normal control

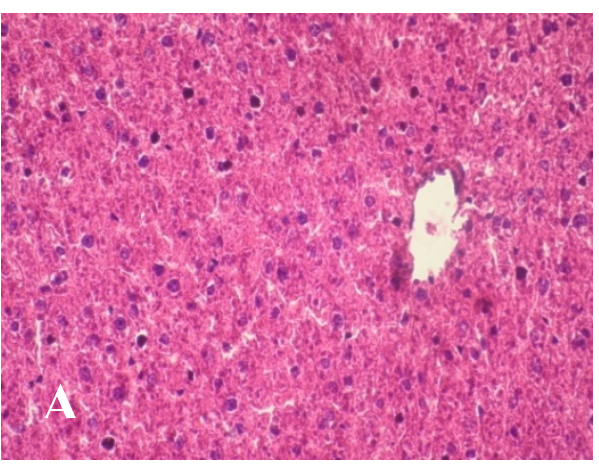

$\mathrm{CCl}_{4}$

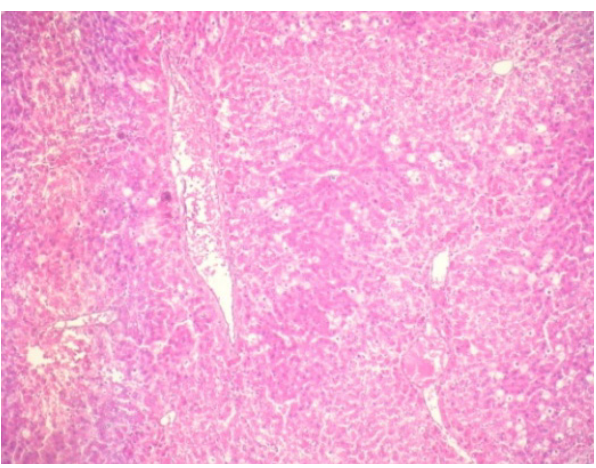

\section{A. gorgonum $(\mathrm{mg} / \mathrm{kg})$} $\mathrm{CCl}_{4}+200$

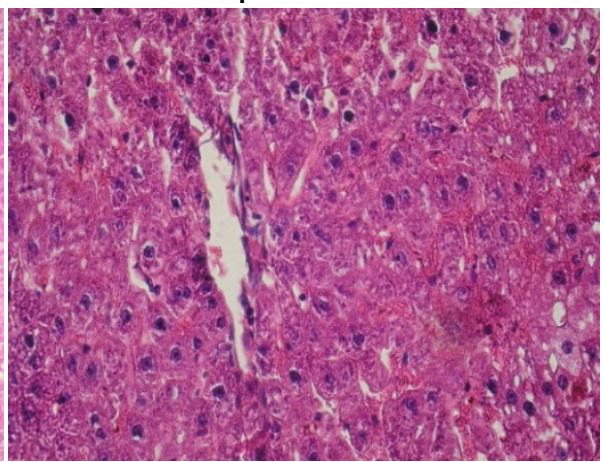

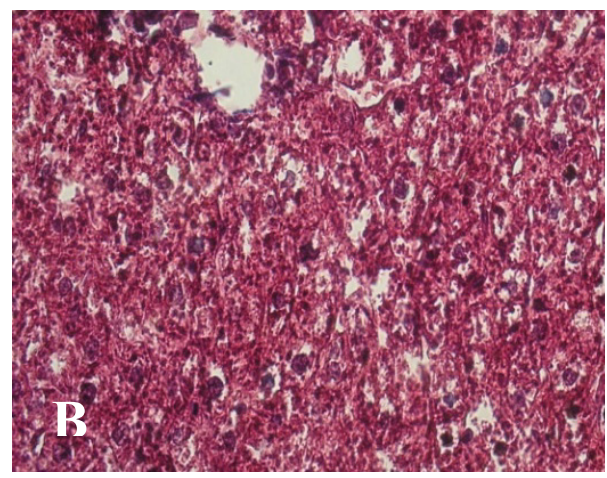
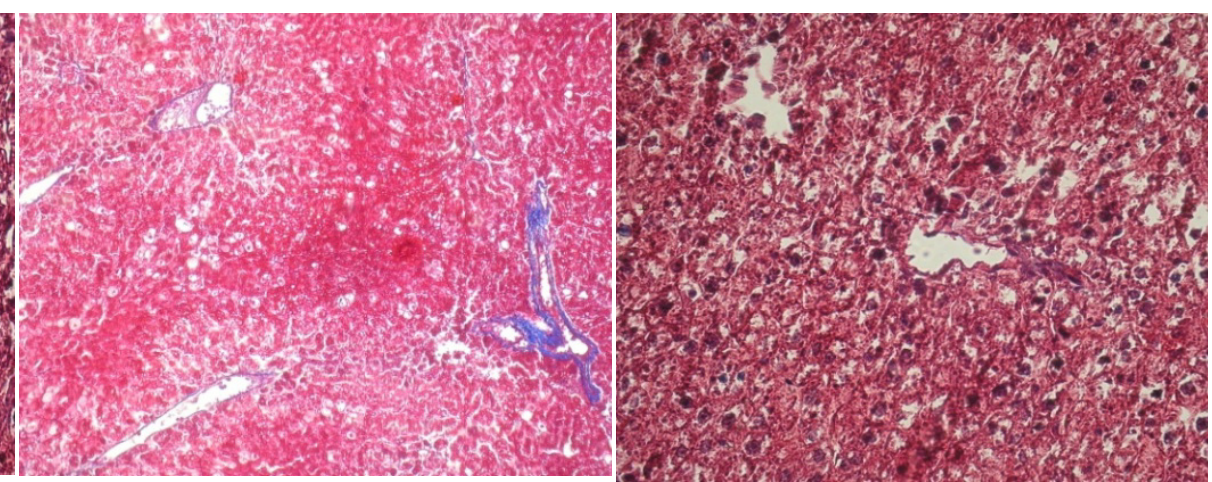

Figure 1. Histomorphological data. The effect of AEAG on liver of $\mathrm{CCl}_{4}$ hepatotoxicity induced rats. The liver tissues were stained with hematoxylin and eosin (A) and Masson's trichrome (B) and then pathophysiologic analysis was performed under light microscopy at $200 \times$ magnification. 